\title{
THE AUTOMORPHISM TOWER OF A FREE GROUP
}

\author{
VLADIMIR TOLSTYKH
}

\begin{abstract}
We prove that the automorphism group of any non-abelian free group $F$ is complete. The key technical step in the proof: the set of all conjugations by powers of primitive elements is first-order parameter-free definable in the group $\operatorname{Aut}(F)$.
\end{abstract}

\section{Introduction}

In 1975 J. Dyer and E. Formanek [2] had proved that the automorphism group of a finitely generated non-abelian free group $F$ is complete (that is, it is centreless and all its automorphisms are inner) and so $\operatorname{Aut}(\operatorname{Aut}(F)) \cong$ $\operatorname{Aut}(F)$. They noted that their research was stimulated by G. Baumslag, who conjectured that the automorphism tower of a finitely generated free group is very short. New proofs for the result of Dyer and Formanek were given in 1990 by D. G. Khramtsov [6] and E. Formanek [4].

The objective of this paper is to generalize the result of Dyer and Formanek from finitely generated non-abelian free groups to arbitrary non-abelian free groups.

Let $F$ be a free non-abelian group. We obtain a group-theoretic characterization of conjugations by powers of primitive elements in $\operatorname{Aut}(F)$. Our key technical results can be summarized in model-theoretic terms as follows: the set of all conjugations by powers of primitive elements is first-order parameter-free definable in the group $\operatorname{Aut}(F)$ (Theorem 5.1). The latter means that there is a first-order formula with one free variable in the language of groups such that its realizations in $\operatorname{Aut}(F)$ are exactly conjugations just mentioned. Therefore the subgroup of all conjugations (inner automorphisms of $F$ ) is a characteristic subgroup of $\operatorname{Aut}(F)$. This implies that the group $\operatorname{Aut}(F)$ is complete (Theorem 5.4).

The main technical tool in the proof of Theorem 5.1 is the use of conjugacy classes of involutions based on a characterization of involutions in $\operatorname{Aut}(F)$ given by J. Dyer and G. P. Scott in [3]. An important role is played by involutions of the following sort. Let $x$ be a primitive element of $F$ and $F=\langle x\rangle * C$ a free factorization of $F$. Then an automorphism of $F$ which inverts $x$ and takes each element in $C$ to its conjugate by $x$, is an involution. We call any involution obtained in such a way a quasi-conjugation, since it acts as conjugation on a 'large' subgroup of $F$.

Date: September 19, 1997.

1991 Mathematics Subject Classification. 20F28 (20E05, 03C60).

Supported by Russian Foundation of Fundamental Research Grant 96-01-00456. 
Suppose $\operatorname{rank} F>2$. For given a quasi-conjugation $\varphi \in \operatorname{Aut}(F)$ let $\Pi$ be the set of all automorphisms of $F$ of the form $\sigma \sigma^{\prime}$, where $\sigma$ and $\sigma^{\prime}$ both commute with $\varphi$ and are conjugate. We prove (Proposition 5.3) that if $\varphi$ is defined by $x$ and $C$ then conjugations by powers of $x$ are exactly the members of the centralizer of $\Pi$ in $\operatorname{Aut}(F)$ which are not involutions. Similarly, we characterize conjugations by powers of primitive elements in the case when $\operatorname{rank} F=2$ (Proposition 5.2). These results reduce the problem of first-order characterization of conjugations by powers of primitive elements to a characterization of quasi-conjugations. The latter problem is solved in Section 4 : we characterize the class of all quasi-conjugations in terms of products of conjugacy classes.

We say that a subset of a group is anti-commutative if its elements are pairwise non-commuting. In the case when $\operatorname{rank} F>2$ we prove that the class of all quasi-conjugations is the unique anti-commutative conjugacy class of involutions such that, for every anti-commutative conjugacy class $K^{\prime}$ of involutions, all involutions in $K K^{\prime}$ are conjugate (Proposition 4.6). When $\operatorname{rank} F=2$ the class of all quasi-conjugations is the unique anti-commutative conjugacy class $K$ of involutions such that elements in $K$ are not squares (Proposition 4.5). It enables us to construct a first-order formula characterizing quasi-conjugations in $\operatorname{Aut}(F)$, and hence to do the same for conjugations by powers of primitive elements in $F$.

The results on conjugacy classes of involutions in $\operatorname{Aut}(F)$ needed in the main body of the paper are considered in Sections 2 and 3 .

The author is very grateful to his colleagues in Kemerovo University Oleg Belegradek, Valery Mishkin, and Peter Biryukov for reading of the first draft of this paper and helpful comments. The main result of the paper (Theorem 5.4) was announced in the abstract [1].

\section{Notation and preliminaries}

In what follows $F$ stands for a free non-abelian group. The free abelian group $F /[F, F]$ of the same rank is denoted by $A$. The natural homomorphism $w \mapsto \bar{w}$ from the group $F$ to $A$ provides the homomorphism $\operatorname{Aut}(F) \rightarrow \operatorname{Aut}(A)$. To denote this homomorphism we shall be using the same symbol - . We shall also say that an automorphism $\varphi$ of $F$ induces the automorphism $\bar{\varphi} \in \operatorname{Aut}(A)$.

We shall use the following fact.

Proposition 1.1. ([10]) Let $G$ be a centreless group. Then the group $\operatorname{Aut}(G)$ is complete if and only if the subgroup $\operatorname{Inn}(F)$ of inner automorphisms (conjugations) is characteristic in $\operatorname{Aut}(G)$.

In this paper we prefer to call elements in the subgroup $\operatorname{Inn}(F)$ 'conjugations' rather than 'inner automorphisms of $F$ '.

It is convenient to formulate our main technical results using a model-theoretic notion of definable set (see [5, ch. II]). An $n$-ary relation $S$ on a group $G$ is said to be first-order definable without parameters in $G$ (or, for short parameterfree definable $)$ if there is a first-order formula $\chi\left(v_{1}, \ldots, v_{n}\right)$ in the language of groups $\left\{\cdot,{ }^{-1}, 1\right\}$ such that $S$ is the set of all $n$-tuples $\left(a_{1}, \ldots, a_{n}\right)$ in $G$ realizing $\chi\left(a_{1}, \ldots, a_{n}\right)$ in the group $G$. For example, the centre of $G$ is parameter-free 
definable by the formula $(\forall u)(v u=u v)$. Clearly, every parameter-free definable relation on $G$ admits a description in terms of the group operation. Therefore

Proposition 1.2. Any parameter-free definable subset of a group $G$ is invariant under all automorphisms of $G$ and hence generates a characteristic subgroup of $G$.

We shall prove that for any non-abelian free group $F$ the set of all quasiconjugations and the set of all conjugations by powers of primitive elements both are parameter-free definable in $\operatorname{Aut}(F)$ and hence are invariant under automorphisms of $\operatorname{Aut}(F)$.

\section{Involutions}

In [3] J. Dyer and G. P. Scott obtained a description of automorphisms of $F$ of prime order. For involutions that description yields the following

Theorem 2.1. [3, p. 199] For every involution $\varphi$ in the group $\operatorname{Aut}(F)$ there is a basis $\mathcal{B}$ of $F$ of the form

$$
\{u: u \in U\} \cup\left\{z, z^{\prime}: z \in Z\right\} \cup\left\{x, y: x \in X, y \in Y_{x}\right\}
$$

on which $\varphi$ acts as follows

$$
\begin{gathered}
\varphi u=u, \quad u \in U, \\
\left\{\begin{array}{l}
\varphi z=z^{\prime} \quad z \in Z, \\
\varphi z^{\prime}=z, \\
\begin{cases}\varphi x=x^{-1}, & x \in X, \\
\varphi y=x y x^{-1}, & y \in Y_{x} .\end{cases}
\end{array}\right.
\end{gathered}
$$

Specifically, the fixed point subgroup of $\varphi, \operatorname{Fix}(\varphi)$, is the subgroup $\langle u: u \in U\rangle$, and hence is a free factor of $F$.

We shall call a basis of $F$ on which $\varphi$ acts similar to (2.1) a canonical basis for $\varphi$. In view of (2.1) one can partition every canonical basis $\mathcal{B}$ for $\varphi$ as follows

$$
\mathcal{B}=U(\mathcal{B}) \cup Z(\mathcal{B}) \cup\left\{z^{\prime}: z \in Z(\mathcal{B})\right\} \cup X(\mathcal{B}) \cup \bigcup_{x \in X(\mathcal{B})} Y_{x}(\mathcal{B}) .
$$

We shall also call any set of the form $\{x\} \cup Y_{x}$, where $x \in X(\mathcal{B})$ a block of $\mathcal{B}$, and the cardinal $\left|Y_{x}\right|+1$ the size of a block. The subgroup generated by the set $Y_{x}$ will be denoted by $C_{x}$ ( $\varphi$ operates on this subgroup as conjugation by $x)$, and the subgroup generated by the block $\{x\} \cup Y_{x}$ will be denoted by $H_{x}$. Sometimes we shall be using more 'accurate' notation like $C_{x}^{\varphi}$ or $H_{x}^{\varphi}$. The set $U(\mathcal{B})$ will be called the fixed part of $\mathcal{B}$.

Clearly, if $\mathcal{B}$ and $\mathcal{C}$ are some canonical bases for involutions $\varphi, \psi$, respectively, and the action of $\varphi$ on $\mathcal{B}$ is isomorphic to the action of $\psi$ on $\mathcal{C}$ (that is the corresponding parts of their canonical bases given by (2.2) are equipotent), symbolically $\varphi|\mathcal{B} \cong \psi| \mathcal{C}$, then $\varphi$ and $\psi$ are conjugate.

For the sake of simplicity we prove the converse (in fact a stronger result) only for involutions we essentially use: for involutions with $Z(\mathcal{B})=\varnothing$ in all canonical 
bases $\mathcal{B}$ 's. We shall call these involutions soft involutions. It is useful that involutions in $\operatorname{Aut}(A)$ induced by them have a sum of eigen \pm -subgroups equal to $A$, like involutions in general linear groups over division rings of characteristic $\neq 2$.

We shall say that involutions $\varphi, \psi \in \operatorname{Aut}(F)$ have the same canonical form, if for all canonical bases $\mathcal{B}, \mathcal{C}$ of $\varphi$ and $\psi$, respectively, $\varphi|\mathcal{B} \cong \psi| \mathcal{C}$. Note that $a$ priori we cannot even claim that the relation we introduce is reflexive.

Proposition 2.2. Let $\varphi \in \operatorname{Aut}(F)$ be a soft involution. An involution $\psi \in$ $\operatorname{Aut}(F)$ is conjugate to $\varphi$ if and only if $\psi$ is soft and $\varphi, \psi$ have the same canonical form.

Proof. Let $A_{2}=A / 2 A$ that is the quotient group of $A$ by the subgroup of even elements. Natural homomorphisms $F \rightarrow A$ and $A \rightarrow A_{2}$, gives us a homomorphism $\mu: \operatorname{Aut}(F) \rightarrow \operatorname{Aut}\left(A_{2}\right)$. Clearly, by 2.1 the family of all involutions in ker $\mu$ coincides with the family of all soft involutions. Therefore an involution which is conjugate to a soft involution is soft too.

Lemma 2.3. Let $\varphi$ be a soft involution with a canonical basis $\mathcal{B}$.

(i) Suppose $a$ is an element in $F$ such that $\varphi a=a^{-1}$. Then $a=\varphi(w) w^{-1}$ or $a=\varphi(w) x w^{-1}$ for some $x \in X=X(\mathcal{B})$ and $w \in F$.

(ii) Suppose $C$ is a maximal subgroup of $F$ on which $\varphi$ acts as conjugation by $x \in X(\mathcal{B})$ :

$$
C=\left\{c \in F: \varphi(c)=x c x^{-1}\right\}
$$

Then $C=C_{x}^{\varphi}=\left\langle Y_{x}\right\rangle$.

Proof. (i) By 2.1 we have

$$
F=\operatorname{Fix}(\varphi) * \prod_{x \in X}^{*} H_{x}
$$

where each factor is $\varphi$-invariant. Then $a=a_{1} \ldots a_{n}$, where every $a_{i}, i=1, \ldots, n$ is an element of a free factor in expansion (2.3), and $a_{i}$ and $a_{i+1}$ lie in different factors for every $i=1, \ldots, n-1$ (that is the sequence $a_{1}, a_{2}, \ldots, a_{n}$ is reduced). Hence if $\varphi(a)=a^{-1}$, or $\varphi\left(a_{1}\right) \ldots \varphi\left(a_{n}\right) a_{1} \ldots a_{n}=1$ then

$$
\varphi\left(a_{n}\right) a_{1}=1, \quad \varphi\left(a_{n-1}\right) a_{2}=1, \quad \ldots
$$

Therefore $a=\varphi\left(w_{0}\right) w_{0}{ }^{-1}$ or $a=\varphi\left(w_{0}\right) v w_{0}{ }^{-1}$, where $v \in H_{x}$ for some $x \in X$ and $\varphi(v)=v^{-1}$.

So let us prove, using induction on length of a word $v$ in the basis $\mathcal{B}$, that $v=\varphi\left(w_{1}\right) w_{1}^{-1}$ or $v=\varphi\left(w_{1}\right) x w_{1}^{-1}$. The only words $v$ of length one in $H_{x}$ with $\varphi v=v^{-1}$ are $x$ and $x^{-1}=\varphi(x) x x^{-1}$.

An arbitrary element $v \in H_{x}$ can be written in the form

$$
v=x^{k_{1}} y_{1} x^{k_{2}} y_{2} \ldots x^{k_{m}} y_{m},
$$

where $y_{i} \in C_{x}$, the elements $x^{k_{1}}$ and $y_{m}$ could be equal to 1 , but any other element is non-trivial. Since $\varphi$ acts on $C_{x}$ as conjugation by $x$ we have

$$
\varphi(v)=x^{-k_{1}+1} y_{1} x^{-k_{2}} y_{2} \ldots x^{-k_{m}} y_{m} x^{-1} .
$$


Suppose that $\varphi(v) v=1$. We then have

$$
x^{-k_{1}+1} y_{1} x^{-k_{2}} y_{2} \ldots x^{-k_{m}} y_{m} x^{-1} x^{k_{1}} y_{1} x^{k_{2}} y_{2} \ldots x^{k_{m}} y_{m}=1 \text {. }
$$

Let first $y_{m} \neq 1$. Then $k_{1}=1$ and $y_{m}=y_{1}{ }^{-1}$. Hence

$$
v=x y_{1} x^{-1}\left(x^{k_{2}+1} y_{2} \ldots x^{k_{m}}\right) y_{1}^{-1}=\varphi\left(y_{1}\right) t y_{1}^{-1} .
$$

It is easy to see that $\varphi(t)=t^{-1}$ and length of $t$ is less than length of $v$. In the case when $y_{m}=1$ we have $k_{m} \neq 0$ and $k_{1}=k_{m}+1$. Therefore,

$$
v=x^{k_{m}}\left(x y_{1} \ldots y_{m-1}\right) x^{k_{m}}=\varphi\left(x^{-k_{m}}\right) t x^{k_{m}},
$$

and we again have that $\varphi(t)=t^{-1}$ and $|t|<|v|$.

(ii) Let $\varphi c=x c x^{-1}$ and $c=c_{1} c_{2} \ldots c_{n}$, where $c_{i}$ are elements in free factors from (2.3) and the sequence $c_{1}, c_{2}, \ldots, c_{n}$ is reduced. Suppose that $n \geq 2$. Due to the $\varphi$-invariance of our free factors, the sequence $\varphi\left(c_{1}\right), \varphi\left(c_{2}\right), \ldots, \varphi\left(c_{n}\right)$ is also reduced and must represent the same element as the sequence $x, c_{1}, c_{2}, \ldots, c_{n}, x^{-1}$. It is easy to see that it is possible if both $c_{1}, c_{n}$ lie in $H_{x}$. In particular, $n \geq 3$. It implies that $\varphi\left(c_{1}\right)=x c_{1}$ and $\varphi\left(c_{n}\right)=c_{n} x^{-1}$. It easily follows from (2.4) and (2.5) there is no $v \in H_{x}$ with $\varphi(v)=x v$; it of course means that for every $v \in H_{x}$ the equality $\varphi(v)=v x^{-1}$ is also impossible, since it is equivalent to $\varphi\left(v^{-1}\right)=x v^{-1}$.

Thus, if $\varphi(c)=x c x^{-1}$, then $c \in H_{x}$. By applying formulae (2.4) and (2.5), one can readily conclude that $c$ must be in $C_{x}$.

Remarks. (a) Note that $a=\varphi(w) w^{-1}$ cannot be a primitive element (i.e. a member of a basis of $F$ ) since $\bar{a}$ is an even element of $A$. Indeed, it follows from (2.3) that

$$
\bar{w}=\overline{w(U)}+\overline{w(X)}+\overline{w(Y)} .
$$

where $w(U) \in \operatorname{Fix}(\varphi), w(X)$ is an element in the subgroup generated by $X$, and $w(Y)$ is an element in the subgroup generated by the set $Y=\bigcup_{x \in X} Y_{x}$. Hence

$$
\begin{aligned}
\bar{a} & =\overline{\varphi(w) w^{-1}}=\overline{\varphi(w)}-\bar{w} \\
& =\overline{(w(U)}-\overline{w(X)}+\overline{w(Y)})-(\overline{w(U)}+\overline{w(X)}+\overline{w(Y)}) \\
& =-2 \overline{w(X)} .
\end{aligned}
$$

(b) Using a similar argument, we see that if $\varphi\left(w_{1}\right) x_{1} w_{1}^{-1}=\varphi\left(w_{2}\right) x_{2} w_{2}^{-1}$, where $x_{1}, x_{2} \in X$, then $x_{1}=x_{2}$ (the element $\bar{x}_{1}-\bar{x}_{2}$ is even if and only if $\left.x_{1}=x_{2}\right)$.

Suppose now that soft involution $\psi$ is a conjugate of $\varphi: \psi=\sigma^{-1} \varphi \sigma$. Let

$$
\mathcal{B}^{\prime}=U^{\prime} \cup X^{\prime} \cup \bigcup_{x^{\prime} \in X^{\prime}} Y_{x^{\prime}}^{\prime}
$$

be a canonical basis for $\psi$. Fixed point subgroups of $\varphi$ and $\psi$ are clearly isomorphic. Thus, $\left|U^{\prime}\right|=|U|$.

If $\psi x^{\prime}=x^{\prime-1}$, where $x^{\prime} \in X^{\prime}$, then $\varphi\left(\sigma x^{\prime}\right)=\left(\sigma x^{\prime}\right)^{-1}$. By 2.3 (i) and the above remarks there is a unique $x \in X$ such that

$$
\sigma x^{\prime}=\varphi(w) x w^{-1}
$$


The mapping $x^{\prime} \mapsto x$ determined in such a way is injective, because otherwise we can find two distinct elements in a basis of a free abelian group whose difference is even.

Hence, $\left|X^{\prime}\right| \leq|X|$ and by symmetry $\left|X^{\prime}\right|=|X|$.

We claim now that

$$
\sigma C_{x^{\prime}}^{\prime}=w C_{x} w^{-1}
$$

It will complete the proof, because in this case $\left|Y_{x^{\prime}}^{\prime}\right|=\left|Y_{x}\right|$.

Let $y^{\prime} \in C_{x^{\prime}}^{\prime}$ and $b=\sigma y^{\prime}$. Since $\psi y^{\prime}=x^{\prime} y^{\prime} x^{\prime-1}$, then $\varphi b=\left(\sigma x^{\prime}\right) b\left(\sigma x^{\prime}\right)^{-1}$. Therefore we have

$$
\varphi\left(w^{-1} b w\right)=\varphi\left(w^{-1}\right) \varphi(b) \varphi(w)=x\left(w^{-1} b w\right) x^{-1} .
$$

Hence by 2.3 (ii) $w^{-1} b w \in C_{x}$.

The equation (2.6) can be rewritten as follows

$$
\sigma^{-1} x=\psi\left(\sigma^{-1} w^{-1}\right) x^{\prime} \sigma^{-1} w
$$

Therefore

$$
\sigma^{-1} C_{x} \subseteq \sigma^{-1} w^{-1} C_{x^{\prime}}^{\prime} \sigma^{-1} w
$$

or

$$
C_{x} \subseteq w^{-1} \sigma C_{x^{\prime}}^{\prime} w
$$

and the result follows.

\section{Anti-commutative conjugacy classes}

The key roles in the group-theoretic characterization of conjugations in $\operatorname{Aut}(F)$ will be played by two conjugacy classes of involutions. First class consists of involutions with a canonical form

$$
\begin{aligned}
& \varphi x=x^{-1}, \\
& \varphi y=x y x^{-1}, y \in Y
\end{aligned}
$$

that is $\mathcal{B}=\{x\} \cup Y$ is a basis of $F$, canonical for $\varphi, U(\mathcal{B})=Z(\mathcal{B})=\varnothing, X(\mathcal{B})$ is a singleton set $\{x\}, Y_{x}=Y$ (any canonical form reproduced below is interpreted in a similar way). We shall call these involutions quasi-conjugations.

An arbitrary element $\varphi \in \operatorname{Aut}(F)$ in the second class has the following canonical form:

$$
\varphi x=x^{-1}, \quad x \in X
$$

that is there is a basis of $F$ such that $\varphi$ inverts all its elements. We shall use for these involutions the term symmetries.

Every symmetry induces in $\operatorname{Aut}(A)$ the automorphism $-\mathrm{id}_{A}$, and hence the product of two symmetries induces $\operatorname{id}_{A}$. Therefore by 2.1 two symmetries commute if and only if they are coincide. Thus, the conjugacy class of all symmetries is, say, anti-commutative, since its elements are pairwise non-commuting. In the next section we shall prove that the class of all quasi-conjugation is also anti-commutative.

In order to characterize conjugations we shall use anti-commutative conjugacy classes of involutions, but we need not an exact determination of all such 
conjugacy classes: it suffices to know that they lie in some 'easy-to-define' family. In the following proposition we formulate and prove a necessary condition of being an anti-commutative conjugacy class, but do not prove its sufficiency.

Proposition 3.1. Let $\varphi$ be an involution in an anti-commutative conjugacy class. Then either $\varphi$ has a canonical form such that

$$
\begin{aligned}
& \varphi u=u, \quad u \in U, \\
& \varphi x=x^{-1}, \quad x \in X, \\
& \varphi y=x y x^{-1}, \quad y \in Y_{x},
\end{aligned}
$$

where $|X| \geq 2$, all the sets $Y_{x}, x \in X$ have the same finite power $n$ and $|U|<$ $n+1$, or $\varphi$ has a canonical form such that

$$
\begin{array}{ll}
\varphi u & =u, \quad u \in U, \\
\varphi x & =x^{-1}, \\
\varphi y & =x y x^{-1}, \quad y \in Y,
\end{array}
$$

where the cardinal $|U|$ is finite and less than $|Y|+1$.

In other words, in the terminology introduced in Section 2, a canonical basis $\mathcal{B}$ for an involution in an anti-commutative conjugacy class either contains exactly one block and the power of the fixed part of $\mathcal{B}$ is less than the size of this block, or all blocks of $\mathcal{B}$ have the same finite size and the power of the fixed part is less than the size of any block. Clearly, symmetries have the form (3.1) (all blocks of their canonical bases have the size one), and quasi-conjugations have the form (3.1) (the size of the unique block is equal to $\operatorname{rank} F$ ).

Proof. Show first that every anti-commutative class of involutions consists only of soft involutions. Indeed, let involution $\varphi$ be an involution whose canonical basis $\mathcal{B}$ has non-empty 'permutational' part $Z(\mathcal{B})$. Suppose $\varphi$ takes $z \in Z(\mathcal{B})$ to $z^{\prime}$. Consider an involution $\psi$ which acts on $\mathcal{B} \backslash\left\{z, z^{\prime}\right\}$ exactly as $\varphi$ does, but taking $z$ to $z^{\prime-1}$. Clearly, $\psi$ is conjugate to $\varphi$ and commutes with $\varphi$.

The following example demonstrates why the size of the fixed part of a canonical basis must necessarily be less than the size of each block:

$$
\left\{\begin{array} { l } 
{ \varphi x = x ^ { - 1 } , } \\
{ \varphi y = x y x ^ { - 1 } , } \\
{ \varphi x _ { 1 } = x _ { 1 } , } \\
{ \varphi y _ { 1 } = y _ { 1 } , } \\
{ \varphi u = u }
\end{array} \quad \left\{\begin{array}{l}
\psi x=x \\
\psi y=y \\
\psi x_{1}=x_{1}{ }^{-1} \\
\psi y_{1}=x_{1} y_{1} x_{1}^{-1} \\
\psi u=u .
\end{array}\right.\right.
$$

Let us make a technical remark. Involutions

$$
\left\{\begin{array} { l } 
{ \varphi x = x ^ { - 1 } , } \\
{ \varphi y = x y x ^ { - 1 } , y \in Y }
\end{array} \text { and } \left\{\begin{array}{l}
\psi x=x^{-1}, \\
\psi y=x^{-1} y x^{-1}, y \in Y
\end{array}\right.\right.
$$

are conjugate: the second one acts 'canonically' on the set $\left\{x, x^{-1} y: y \in Y\right\}$ : $\psi\left(x^{-1} y\right)=x\left(x^{-1} y\right) x^{-1}$. 
Let now $\varphi$ be a soft involution with a canonical basis $\mathcal{B}$ such that for some distinct $x_{1}, x_{2} \in X(\mathcal{B})$ either $\left|Y_{x_{1}}\right|<\left|Y_{x_{2}}\right|$ or $\left|Y_{x_{1}}\right|=\left|Y_{x_{2}}\right|$ and the cardinal $\left|Y_{x_{1}}\right|$ is infinite (thus, there must be no neither a pair of blocks of different size nor a pair of infinite blocks). The fact that $\varphi$ commutes with at least two its conjugates is a consequence of (3.1) and the following

Claim 3.2. Let $G$ be a free group with a basis

$$
\{x, a\} \cup B \cup\{c\} \cup D \cup E,
$$

where $|B|=|D|$. Then

(i) Involutions

$$
\left\{\begin{array} { l } 
{ \varphi x = x ^ { - 1 } , } \\
{ \varphi a = x ^ { - 1 } a x ^ { - 1 } , } \\
{ \varphi b = x ^ { - 1 } b x ^ { - 1 } , } \\
{ \varphi c = c ^ { - 1 } , } \\
{ \varphi d = c ^ { - 1 } d c ^ { - 1 } , } \\
{ \varphi e = x ^ { - 1 } e x ^ { - 1 } }
\end{array} \quad \left\{\begin{array}{l}
\psi x=x^{-1} \\
\psi a=a^{-1} \\
\psi b=a^{-1} b a^{-1}, b \in B \\
\psi c=x^{-1} c x^{-1} \\
\psi d=x^{-1} d x^{-1}, d \in D \\
\psi e=x^{-1} e x^{-1}, e \in E
\end{array}\right.\right.
$$

are conjugate and commute.

(ii) Furthermore,

$$
\begin{aligned}
& \operatorname{rank} C_{x}^{\varphi}=|\{a\} \cup B \cup E|=|B|+|E|+1, \\
& \operatorname{rank} C_{c}^{\varphi}=|D| .
\end{aligned}
$$

Thus, $\operatorname{rank} C_{c}^{\varphi} \leq \operatorname{rank} C_{x}^{\varphi}$, where equality holds only if both ranks are infinite.

Proof. Easy.

To complete the proof of the Proposition, we should cut off involutions with a canonical form such that

$$
\begin{aligned}
& \varphi u=u, \quad u \in U, \\
& \varphi x=x^{-1}, \\
& \varphi y=x y x^{-1}, \quad y \in Y,
\end{aligned}
$$

where $|U|$ is infinite. To do this let us consider involutions $\varphi, \psi$ which act on a basis $U \cup\{x\} \cup Y$ of $F$ as follows

$$
\left\{\begin{array} { l } 
{ \varphi u _ { 0 } = u _ { 0 } , } \\
{ \varphi u = u , } \\
{ \varphi x = x ^ { - 1 } , } \\
{ \varphi y = x y x ^ { - 1 } , }
\end{array} \quad \left\{\begin{array}{l}
\psi u=u_{0}^{-1}, \\
\psi u=u, \\
\psi x=u_{0} x u_{0}^{-1}, \\
\psi y=u_{0} y u_{0}^{-1}, \quad y \in Y,
\end{array}\right.\right.
$$

where $u_{0}$ is a fixed element in $U$. It is readily seen that $\varphi$ and $\psi$ are conjugate and commute. 


\section{Quasi-conjugations}

In this section we obtain a (first-order) characterization of quasi-conjugations in $\operatorname{Aut}(F)$. First we prove that the conjugacy class of all quasi-conjugations is anti-commutative. Then we distinguish this class from other anti-commutative conjugacy classes of involutions: it is trivial in the case when $\operatorname{rank} F=2$ and more technical in the case when $\operatorname{rank} F>2$.

Proposition 4.1. The class of all quasi-conjugation is an anti-commutative conjugacy class.

Proof. Let $\varphi$ be a quasi-conjugation with a canonical basis $\mathcal{B}=\{x\} \cup Y$ :

$$
\begin{aligned}
& \varphi x=x^{-1}, \\
& \varphi y=x y x^{-1}, y \in Y
\end{aligned}
$$

Every $\sigma \in \operatorname{Aut}(F)$, which commutes with $\varphi$, takes $x$ to a primitive element of the form $\varphi(w) x w^{-1}, w \in F$ (Lemma 2.3). It turns out that the only primitive elements of the form $\varphi(w) x w^{-1}$ are $x$ and $x^{-1}$. This fact is a consequence of the following result, which we shall use once more later.

Lemma 4.2. Let $\alpha$ be an involution with a canonical form such that

$$
\begin{aligned}
& \alpha x=x^{-1}, \\
& \alpha y=x y x^{-1}, \quad y \in Y=Y_{x}, \\
& \alpha u=u, \quad u \in U,
\end{aligned}
$$

and $a \in F$ a primitive element, which $\alpha$ sends to its inverse. Then $a=v x^{ \pm 1} v^{-1}$, where $v \in\langle U\rangle=\operatorname{Fix}(\alpha)$.

Proof. As we observed earlier $a=\alpha(w) x w^{-1}$. It is easy to see that $a$ lies in the normal closure of $x$. One can use induction on length of a reduced word $w$ in the basis $\{x\} \cup Y \cup U$. We have

$$
\begin{aligned}
& \alpha\left(x w_{1}\right) x w_{1}^{-1} x^{-1}=x^{-1}\left(\alpha\left(w_{1}\right) x w_{1}^{-1}\right) x^{-1}, \\
& \alpha\left(u w_{1}\right) x w_{1}^{-1} u^{-1}=u\left(\alpha\left(w_{1}\right) x w_{1}^{-1}\right) u^{-1}, \\
& \alpha\left(y w_{1}\right) x w_{1}^{-1} y^{-1}=x \cdot y\left(x^{-1} \alpha\left(w_{1}\right) x w_{1}^{-1}\right) y^{-1} .
\end{aligned}
$$

Proposition . (『8], [7, II.5.15]) Let $F$ be a free group and the normal closure of $q \in F$ consists of a primitive element $p$. Then $q$ is conjugate to $p$ or $p^{-1}$.

Hence $a=b x^{\varepsilon} b^{-1}$, where $\varepsilon= \pm 1$. Since $\alpha(a)=a^{-1}$, we have

$$
\alpha(b) x^{-\varepsilon} \alpha\left(b^{-1}\right)=b x^{-\varepsilon} b^{-1} .
$$

It follows that $x^{-\varepsilon}$ and $b^{-1} \alpha(b)$ commute. Therefore both these elements lie in a cyclic subgroup of $F$ ([0, I.2.17]). It must be the subgroup $\langle x\rangle$, because $x$ is primitive. Hence $\alpha(b)=b x^{k}$. If $k$ is even, say $k=2 m$, then $\alpha\left(b x^{m}\right)=b x^{m}$ and $v=b x^{m} \in\langle U\rangle$. In the case when $k$ is odd, we have there is $z \in F$ such that $\alpha(z)=z x$. One can easily check that this is impossible (e.g. using 'abelian' arguments as in Section (2). 
Proposition 4.3. The centralizer of a quasi-conjugation $\varphi$ with a canonical basis $\{x\} \cup Y$ consists of automorphisms $\sigma \in \operatorname{Aut}(F)$ of the form

$$
\begin{aligned}
& \sigma x=x, \\
& \sigma y=\theta(y), y \in Y,
\end{aligned}
$$

and of the form

$$
\begin{aligned}
& \sigma x=x^{-1}, \\
& \sigma y=x \theta(y) x^{-1}, y \in Y,
\end{aligned}
$$

where $\theta \in \operatorname{Aut}\left(C_{x}\right)$.

Proof. The Proposition easily follows from Lemma 4.2 and one more

Lemma 4.4. [1, p. 101] Let $F=G * H$ be a free factorization of a free group F. Suppose that $\alpha \in \operatorname{Aut}(F)$ and $\left.\alpha\right|_{G}$ is an endomorphism of $G$. Then $\left.\alpha\right|_{G} \in$ $\operatorname{Aut}(G)$ if either $\operatorname{rank} G$ or $\operatorname{rank} H$ is finite.

By 4.2 if $\sigma \in \operatorname{Cen}(\varphi)$, then either $\sigma x=x$ or $\sigma x=x^{-1}$. Assume that $\sigma$ fixes $x$. Then, for each $y \in C_{x}$

$$
\varphi \sigma y=\sigma x \sigma y \sigma x^{-1}=x \sigma y x^{-1} .
$$

Therefore $\sigma y \in C_{x}$.

If $\sigma x=x^{-1}$, then $\varphi \sigma x=x$. It follows that $\varphi \sigma$ has the form (4.1), and hence $\sigma$ must have the form (4.1).

We can prove now that $\varphi$ is the unique quasi-conjugation in its centralizer, or, in other words, the conjugacy class of $\varphi$ is anti-commutative. Indeed, there are no quasi-conjugations in the family of automorphisms of the form (4.1), because every quasi-conjugation has trivial fixed-point subgroup. Let $\sigma$ be a quasi-conjugation of the form (4.1). Hence $\theta^{2}=\mathrm{id}$, and $\theta$ is either the identity automorphism of $C_{x}$ or a soft involution. Assume that $\theta$ is an involution. By Theorem 2.1 there is a canonical basis $\mathcal{C}$ of a free group $C_{x}$ for $\theta$ such that

$$
\begin{array}{llrl}
\theta u & =u, & & u \in U(\mathcal{C}), \\
\theta c & =c^{-1}, & & c \in X(\mathcal{C}), \\
\theta d & =c d c^{-1}, & & d \in Y_{c}(\mathcal{C}) .
\end{array}
$$

Therefore

$$
\begin{aligned}
& \sigma x=x^{-1}, \\
& \sigma u=x u x^{-1}, \quad u \in U(\mathcal{C}), \\
& \sigma(x c)=(x c)^{-1}, \quad c \in X(\mathcal{C}), \\
& \sigma d=(x c) d(x c)^{-1}, \quad d \in Y_{c}(\mathcal{C}) .
\end{aligned}
$$

We obtain a canonical basis for $\sigma$. This basis contains at least two blocks, and hence by Proposition 2.2 $\sigma$ cannot be a quasi-conjugation. So $\theta$ must be the identity automorphism, or, equivalently, $\sigma=\varphi$ as desired. This completes the proof. 
Now there are no further problems in a characterization of quasi-conjugations in automorphism groups of two-generator free groups.

Proposition 4.5. Suppose that $\operatorname{rank} F=2$. Then the class of all quasi-conjugations is the unique anti-commutative conjugacy class $K$ of involutions such that elements in $K$ are not squares.

Proof. By Propositions 3.1 and 4.1 the only anti-commutative conjugacy classes of involutions in $\operatorname{Aut}(F)$ are the class of all quasi-conjugations and the class of all symmetries. Every symmetry is a square:

$$
\left\{\begin{array} { l } 
{ \psi x _ { 1 } = x _ { 2 } { } ^ { - 1 } } \\
{ \psi x _ { 2 } = x _ { 1 } }
\end{array} \Rightarrow \left\{\begin{array}{l}
\psi^{2} x_{1}=x_{1}{ }^{-1} \\
\psi^{2} x_{2}=x_{2}{ }^{-1}
\end{array}\right.\right.
$$

On the other hand, a quasi-conjugation $\varphi$ with a canonical form

$$
\begin{aligned}
& \varphi x=x^{-1}, \\
& \varphi y=x y x^{-1}
\end{aligned}
$$

induces in $\operatorname{Aut}(A)$ an automorphism with the determinant which is equal to -1 . Hence $\bar{\varphi}$ cannot be a square in $\operatorname{Aut}(A)$. It implies that $\varphi$ is not a square in $\operatorname{Aut}(F)$.

Let us consider now more serious case.

Theorem 4.6. Let $\operatorname{rank} F>2$. Then the class of all quasi-conjugations is the unique anti-commutative conjugacy class $K$ of involutions such that for every anti-commutative conjugacy class $K^{\prime}$ of involutions, all involutions in $K K^{\prime}$ are conjugate.

Proof. Let $\varphi$ be a quasi-conjugation, $K^{\prime}$ an anti-commutative conjugacy class of involutions, and $\varphi \notin K^{\prime}$. It suffices to prove that if $\psi, \psi^{\prime} \in K^{\prime}$ both commute with $\varphi$, then $\varphi \psi$ and $\varphi \psi^{\prime}$ are conjugate.

Suppose first that fixed point subgroups of $\psi$ and $\psi^{\prime}$ are trivial. Then both $\psi$ and $\psi^{\prime}$ have the form (4.1):

$$
\begin{array}{ll}
\psi x=x^{-1}, & \psi^{\prime} x=x^{-1} \\
\psi y=x \theta(y) x^{-1}, & \psi^{\prime} y=x \theta^{\prime}(y) x^{-1}, \quad y \in Y,
\end{array}
$$

where $\theta$ and $\theta^{\prime}$ are in $\operatorname{Aut}\left(C_{x}\right)$. It is easy to see that $\theta$ and $\theta^{\prime}$ are soft involutions. Let $\mathcal{C}$ be a canonical basis for $\theta$. As we observed above, the set

$$
(\{x\} \cup U(\mathcal{C})) \cup \bigcup_{c \in X(\mathcal{C})}\left(\{x c\} \cup Y_{c}(\mathcal{C})\right)
$$

is a canonical basis for $\psi$, and (4.1) is a partition of this basis into blocks (there are at least two blocks, because $\psi$ cannot be conjugate to $\varphi$ ). Since $\psi$ lies in an anti-commutative conjugacy class, all these blocks have the same size, and hence for all $c \in X(\mathcal{C})$

$$
|U(\mathcal{C})|=\left|Y_{c}(\mathcal{C})\right|
$$

By applying a similar argument to $\psi^{\prime}$, we see that $\theta$ and $\theta^{\prime}$ are conjugate in $\operatorname{Aut}\left(C_{x}\right)$. Hence $\varphi \psi$ and $\varphi \psi^{\prime}$ are conjugate in $\operatorname{Aut}(F)$ : both these automorphisms fix $x$ and their restrictions on $C_{x}\left(\theta\right.$ and $\left.\theta^{\prime}\right)$ are conjugate. 
Assume that $\psi$ and $\psi^{\prime}$ have non-trivial fixed point subgroups. Both $\psi$ and $\psi^{\prime}$ preserve the subgroup $C_{x}$ and fix the element $x$. It means (Propositions 2.1, 2.2) that restrictions of $\psi$ and $\psi^{\prime}$ on $C_{x}$, say, $\theta$ and $\theta^{\prime}$, respectively are conjugate in $\operatorname{Aut}\left(C_{x}\right): \theta^{\prime}=\pi^{-1} \theta \pi$, where $\pi \in \operatorname{Aut}\left(C_{x}\right)$. The automorphism $\pi$ can be extended to an element $\sigma \in \operatorname{Aut}(F)$ such that $\sigma x=x$. By $4.3 \sigma$ commutes with $\varphi$, and hence

$$
\sigma^{-1}(\varphi \psi) \sigma=\varphi \psi^{\prime}
$$

Let us prove the converse. We start with the following

Claim 4.7. Assume that an involution $\varphi \in \operatorname{Aut}(F)$ is an element of an anticommutative conjugacy class, and $\varphi$ is neither quasi-conjugation, nor symmetry. Then there exist symmetries $\psi$ and $\psi^{\prime}$ such that $\varphi \psi$ and $\varphi \psi^{\prime}$ are nonconjugate involutions.

Proof. (a) A natural way to define an action of a symmetry commuting with $\varphi$ on a block of a canonical basis for $\varphi$ is given by Proposition 4.3:

$$
\begin{array}{ll}
\varphi x=x^{-1}, & \psi x=x^{-1} \\
\varphi y=x y x^{-1} & \psi y=x y^{-1} x^{-1}, \quad\left(\psi(x y)=(x y)^{-1}\right) \quad y \in Y_{x} .
\end{array}
$$

Clearly, the product of $\varphi$ and $\psi$ fixes $x$ and inverts each element in $Y_{x}$.

(b) A way to define an action of a symmetry on the fixed part of a canonical basis is obvious: a symmetry should invert each element.

If a symmetry $\psi$ acts on a canonical basis for $\varphi$ as it is defined in (a) and (b), then any canonical basis of the product $\varphi \psi$ has non-empty fixed part and each block of this basis has the size one.

(c) Assume now that a canonical basis $\mathcal{B}$ for $\varphi$ contains at least two blocks, say,

$$
(\{x\} \cup(\{y\} \cup B)) \text { and }(\{z\} \cup(\{t\} \cup C))
$$

Hence

$$
\begin{aligned}
& \varphi x=x^{-1}, \\
& \varphi y=x y x^{-1}, \\
& \varphi a=x b x^{-1}, \quad b \in B, \\
& \varphi z=z^{-1}, \\
& \varphi t=z t z^{-1}, \\
& \varphi c=z c z^{-1}, \quad c \in C
\end{aligned}
$$

Then we can choose a symmetry $\psi$ such that any canonical basis for $\varphi \psi$ has a block of the size two. Let $\mathcal{C}$ denote the union of blocks in (4.2). Suppose that $\psi$ acts on $\mathcal{B} \backslash \mathcal{C}$ as it is defined in (a) and (b), and define its action on the 
subgroup generated by $\mathcal{C}$ as follows

$$
\begin{aligned}
& \psi(t x)=(t x)^{-1}, \\
& \psi y \quad=(t x) y^{-1}(t x)^{-1}, \\
& \psi b \quad=(t x) b^{-1}(t x)^{-1}, \quad b \in B \\
& \psi z \quad=z^{-1} \\
& \psi t \quad=z t^{-1} z^{-1}, \\
& \psi c \quad=z c^{-1} z^{-1}, \quad c \in C
\end{aligned}
$$

Let $\sigma$ denote the automorphism $\varphi \psi$. We have

$$
\sigma(t x)=\varphi\left(x^{-1} t^{-1}\right)=x z t^{-1} z^{-1} .
$$

Since $\sigma z=z$ and $\sigma t=t^{-1}$, then

$$
t^{-1} \sigma(x) z=(x z) t^{-1} \Longleftrightarrow \sigma(x z)=t(x z) t^{-1} .
$$

Furthermore,

$$
\sigma(y)=\varphi\left(t x y^{-1} x^{-1} t^{-1}\right)=\varphi(t) \varphi\left(x y^{-1} x^{-1}\right) \varphi(t)=z t z^{-1} y^{-1} z t^{-1} z^{-1} .
$$

Let $y^{\prime}$ denote the element $z t z^{-1} y$. It easily follows from (4.4) then $\sigma$ inverts $y^{\prime}$. The same is true for all elements $b^{\prime}=z t z^{-1} b$, where $b \in B$. Summing up, we conclude that $\sigma$ is an involution and the basis

$$
(\mathcal{B} \backslash \mathcal{C}) \cup(\{t\} \cup\{x z\}) \cup\left\{y^{\prime}\right\} \cup\left\{b^{\prime}: b \in B\right\} \cup\{z\} \cup C
$$

is a canonical basis for $\sigma$. The formulae in (4.3) demonstrate that this basis contains a block of the size two.

(d) Any canonical basis for $\varphi$ has exactly one block and non-empty fixed part:

$$
\begin{aligned}
& \varphi u=u, \quad u \in U, \\
& \varphi x=x^{-1}, \\
& \varphi y=x y x^{-1}, \quad y \in Y
\end{aligned}
$$

One can easily find a symmetry $\psi$ such that the product $\varphi \psi$ will be nonconjugate to each product of $\varphi$ with a symmetry obtained in a natural way, using (a) and (b):

$$
\begin{array}{ll}
\psi u_{0} & =u_{0}^{-1}, \\
\psi u & =u^{-1}, \\
\psi x & =u_{0} x^{-1} u_{0}^{-1}, \\
\psi y & =u_{0} x y^{-1} x^{-1} u_{0}^{-1}, \quad y \in Y \backslash\left\{u_{0}\right\},
\end{array}
$$

where $u_{0} \in U$. The reason is the same as in the previous point (c): any canonical basis for $\varphi \psi$ contains a block of the size two $\left(\left\{u_{0}\right\} \cup\{x\}\right.$ in this example). 
To complete the proof of the Theorem, we have to find for an arbitrary symmetry $\varphi$ two involutions $\psi, \psi^{\prime}$ from an anti-commutative conjugacy class such that their products with $\varphi$ are non-conjugate involutions. Clearly, the problem is to add one more conjugacy class to the family of definitely known at this moment anti-commutative conjugacy classes of involutions (quasi-conjugations and symmetries). Having such a class, one can rework in an obvious way examples in the proof of the latter Claim, and hence prove the desired result on symmetries. It will complete the proof of Theorem 4.6.

Claim 4.8. Let $\operatorname{rank} F>2$. The conjugacy class of an involution $\psi$ with a canonical form such that

$$
\begin{aligned}
& \psi x=x^{-1}, \\
& \psi y=x y x^{-1}, y \in Y=Y_{x}, \\
& \psi u=u,
\end{aligned}
$$

where $Y \neq \varnothing$ is anti-commutative (the fixed point subgroup of $\psi$ is of rank one).

Proof. Suppose that $\sigma \in \operatorname{Cen}(\psi)$. The only primitive elements which are fixed by $\psi$ are $u$ and $u^{-1}$. Therefore $\sigma u=u^{ \pm 1}$. By Lemma $4.2 \sigma$ must take $x$ to an element of the form $u^{k} x^{ \pm 1} u^{-k}$, where $k \in \mathrm{Z}$. To calculate the image $\sigma y$ of an element $y \in Y$ one may apply the arguments used in the proof of Proposition 4.3. We then have that $\sigma$ has the form

$$
\begin{aligned}
& \sigma u=u^{\varepsilon}, \\
& \sigma x=u^{k} x u^{-k}, \\
& \sigma y=u^{k} \theta(y) u^{-k}, y \in Y,
\end{aligned}
$$

or the form

$$
\begin{aligned}
\sigma u & =u^{\eta}, \\
\sigma x & =u^{m} x^{-1} u^{-m}, \\
\sigma y & =u^{m} x \theta(y) x^{-1} u^{-m}, y \in Y,
\end{aligned}
$$

where $\varepsilon, \eta= \pm 1, k, m$ are integers, and $\theta \in \operatorname{Aut}\left(C_{x}\right)$. Note that if $(\varepsilon=1$ and $k \neq 0)$ or $(\eta=1$ and $m \neq 0)$, then $\sigma$ has infinite order.

Let now $\sigma \in \operatorname{Cen}(\psi)$ be a conjugate of $\psi$. We should prove that $\sigma=\psi$.

Suppose first that $\sigma$ has the form (4.5). When $\varepsilon=1$ and $k=0$, the subgroup $\operatorname{Fix}(\sigma)$ is of rank at least two and $\sigma$ cannot be conjugate to $\psi$, because $\operatorname{rank} \operatorname{Fix}(\psi)=1$. Let $\varepsilon=-1$; then $\theta^{2}=\mathrm{id}$. As we observed above it follows from the assumption $\theta \neq$ id that a canonical basis for $\sigma$ contains at least two blocks. It is impossible. Thus, $\theta=\mathrm{id}$. When $k$ is even, say $k=2 l$, the subgroup $\operatorname{Fix}(\sigma)$ has rank at least two:

$$
\begin{aligned}
\sigma u & =u^{-1}, \\
\sigma\left(u^{l} x u^{-l}\right) & =u^{l} x u^{-l}, \\
\sigma\left(u^{l} y u^{-l}\right) & =u^{l} y u^{-l}, y \in Y,
\end{aligned}
$$


Similarly, we see that in the case when $k$ is odd, $\sigma$ is a quasi-conjugation.

Let us now try to find a conjugate $\sigma$ of $\psi$ in the family of automorphisms of the form (4.5). An involution of this form with $\eta=-1$ is not conjugate to $\psi$ : any canonical basis for such an involution contains more than one block. Thus, $\eta=1$, and we have that $m=0$ and $\theta=\mathrm{id}$. Therefore $\sigma=\psi$.

The proof of Theorem 4.6 is now complete.

Proposition 4.5 and Theorem 4.6 can be summarized in model-theoretic terms as follows.

Theorem 4.9. The set of all quasi-conjugations is first-order parameter-free definable in $\operatorname{Aut}(F)$.

Proof. All the hypotheses in Proposition 4.5 and Theorem 4.6 are in fact firstorder. For instance, the following formula says that the conjugacy class of an involution $v$ is anti-commutative:

$$
\operatorname{ACC}(v)=\left(v \neq 1 \wedge v^{2}=1\right) \wedge(\forall u)\left(\left(v v^{u}\right)^{2}=1 \rightarrow v=v^{u}\right)
$$

where $v^{u}=u v u^{-1}$. There are no difficulties in conversion of other hypotheses into first-order formulae.

REMARKs. (a) In the case when $F$ has infinite rank, a group-theoretic characterization of quasi-conjugations can be obtained in an easier way. Indeed, involutions in anti-commutative conjugacy classes of the form (3.1) (infinitely many finite blocks of the same size in any canonical basis) are in this case squares in $\operatorname{Aut}(F)$ :

$$
\left\{\begin{array} { l } 
{ \sigma x = a ^ { - 1 } , } \\
{ \sigma y = a b a ^ { - 1 } , } \\
{ \sigma a = x , } \\
{ \sigma b = y , }
\end{array} \Rightarrow \left\{\begin{array}{l}
\sigma^{2} x=x^{-1}, \\
\sigma^{2} y=x y x^{-1}, \quad y \in Y \\
\sigma^{2} a=a^{-1}, \\
\sigma^{2} b=a b a^{-1} \quad b \in B \quad(|B|=|Y|) .
\end{array}\right.\right.
$$

On the other hand, it is easy to see that involutions of the form (3.1) (exactly one block in any canonical basis) are not squares. In particular, the condition of being a square distinguishes symmetries from quasi-conjugations. Therefore in order to characterize quasi-conjugation in $\operatorname{Aut}(F)$ one can use the following

Theorem . Let $F$ has infinite rank. Then the class of all quasi-conjugations is the unique anti-commutative conjugacy class $K$ of involutions such that its elements are not squares and for every anti-commutative conjugacy class $K^{\prime}$ of involutions, whose elements are squares, all involutions in $K K^{\prime}$ are conjugate.

A proof of the latter Theorem may follow the plan of the proof of Theorem 4.6, but there is no need to consider the point (c) in the proof of Claim 4.7 and Claim 4.8.

(b) One can easily obtain a uniform first-order characterization of quasi-conjugations. Indeed, the result of S. Meskin [9], [0, I.4.6] states that if $\operatorname{rank} F=$ 2 , then the group $\operatorname{Aut}(F)$ has exactly four conjugacy classes of involutions. The converse is a consequence of Proposition 2.2, since if $\operatorname{rank} F>2$, then $\operatorname{Aut}(F)$ has at least six conjugacy classes of soft involutions (the number of 
conjugacy classes of soft involutions in the group $\operatorname{Aut}\left(F_{3}\right)$, where $F_{3}$ is a threegenerator free group). Suppose that first-order formulae $\mathrm{QC}_{0}(v)$ and $\mathrm{QC}_{1}(v)$ define quasi-conjugations in $\operatorname{Aut}(F)$ in the case when $\operatorname{rank} F=2$ and $\operatorname{rank} F>$ 2 , respectively. Then a first-order formula

$$
\left(\mathrm{QC}_{0}(v) \wedge \chi\right) \vee\left(\mathrm{QC}_{1}(v) \wedge \neg \chi\right),
$$

where a closed first-order formula $\chi$ says about four conjugacy classes of involutions, defines quasi-conjugations in the automorphism group of an arbitrary non-abelian free group.

\section{Conjugations}

The following theorem is our key result in the proof of completeness of $\operatorname{Aut}(F)$.

Theorem 5.1. The set of all conjugations by powers of primitive elements is first-order parameter-free definable in $\operatorname{Aut}(F)$.

Proof. As in the proof of definability of quasi-conjugations we consider two cases: $\operatorname{rank} F=2$ and $\operatorname{rank} F>2$.

I. $F$ is of rank two.

Let $\varphi$ be a quasi-conjugation with a canonical basis $\mathcal{B}=\{x\} \cup\{y\}$. By Proposition 4.3 the centralizer of $\varphi$ consists of four elements. Non-trivial ones are involutions: $\varphi$, a symmetry, and an involution $\psi$ which fixes $x$ and inverts $y$. Clearly, $\psi$ is the unique involution in $\operatorname{Cen}(\varphi)$ commuting with at least two its conjugates.

Proposition 5.2. All conjugations by powers of $x$ are in $\operatorname{Cen}(\psi)$. An element $\sigma$ in $\operatorname{Cen}(\psi)$ is conjugation by a power of $x$ if and only if $\sigma$ is not an involution, and can be represented as the product of two conjugate involutions.

Proof. Let $\sigma \in \operatorname{Cen}(\psi)$. Since $x$ and $x^{-1}$ are the only primitive elements in $\operatorname{Fix}(\psi)$, we have $\sigma x=x^{ \pm 1}$. We can use then Lemma 4.2 (assuming that the size of a block is equal to one). Therefore $\sigma y=x^{k} y^{ \pm 1} x^{-k}$, for some $k \in \mathrm{Z}$. Thus,

$$
\begin{aligned}
& \sigma x=x^{\varepsilon}, \\
& \sigma y=x^{k} y^{\eta} x^{-k},
\end{aligned}
$$

where $\varepsilon, \eta= \pm 1$ (and conversely, every automorphism of $F$ of the latter form commutes with $\psi$ ). In the case when $\varepsilon=-1, \sigma$ is an involution. The automorphism $\sigma$ of $F$ such that

$$
\begin{aligned}
& \sigma x=x, \\
& \sigma y=x^{k} y^{-1} x^{-k}
\end{aligned}
$$

induces in $\operatorname{Aut}(A)$ an automorphism with determinant -1 . On the other hand, the product of two conjugate involutions from $\operatorname{Aut}(F)$ induces in $\operatorname{Aut}(A)$ an automorphism whose determinant is equal to 1 . To complete the proof, we should express as the product of two conjugate involutions an arbitrary conjugation 
by a power of $x$. It is easy:

$$
\begin{array}{llrl}
\alpha x=x^{-1}, & \alpha^{\prime} x=x^{-1} \\
\alpha y=y^{-1}, & \alpha^{\prime} y=x^{-k} y^{-1} x^{k} .
\end{array}
$$

The product of symmetries $\alpha$ and $\alpha^{\prime}$ is evidently conjugation by $x^{k}$.

II. F is of rank at least three.

We also start with a quasi-conjugation $\varphi$. Suppose that $\mathcal{B}=\{x\} \cup Y$ is a canonical basis for $\varphi$. Let $\Pi$ denote the set of all automorphisms of $F$ of the form $\pi=\sigma \sigma^{\prime}$, where $\sigma$ and $\sigma^{\prime}$ are in $\operatorname{Cen}(\varphi)$ and conjugate. By 4.3 conjugate automorphisms $\sigma, \sigma^{\prime}$ in the centralizer of $\varphi$ either both have the form (4.1) (when their fixed point subgroups are non-trivial) or have the form (4.1). Therefore every $\pi \in \Pi$ has the form

$$
\begin{aligned}
& \pi x=x \\
& \pi y=\theta(y), \quad y \in Y,
\end{aligned}
$$

where $\theta \in \operatorname{Aut}\left(C_{x}\right)$, that is $\pi$ fixes $x$ and preserves the subgroup $C_{x}$.

Proposition 5.3. All conjugations by powers of $x$ are in the centralizer of the family $\Pi$. Every member of $\mathrm{Cen}(\Pi)$ is either an involution or conjugation by a power of $x$.

Proof. Let

$$
\mathcal{C}=\{a, b\} \cup C
$$

be a basis of $C_{x}$, and $\tau \in \operatorname{Aut}(F)$ an element of $\operatorname{Cen}(\Pi)$.

First we construct $\pi \in \Pi$ such that the fixed point subgroup of $\pi$ is the subgroup $\langle x, a\rangle$. Since $\tau$ must commute with $\pi$ we shall have that

$$
\tau a=w_{a}(x, a),
$$

where $w_{a}$ is a reduced word in letters $x$ and $a$.

To construct $\pi$, we use the same idea as in the proof of the previous result:

$$
\begin{aligned}
\sigma x & =x, & \sigma^{\prime} x & =x, \\
\sigma a & =a^{-1}, & \sigma^{\prime} a & =a^{-1}, \\
\sigma b & =b^{-1}, & \sigma^{\prime} b & =a^{-1} b^{-1} a, \\
\sigma c & =c^{-1}, & \sigma^{\prime} c & =a^{-1} c^{-1} a, \quad c \in C .
\end{aligned}
$$

The restriction of $\pi=\sigma \sigma^{\prime}$ on $C_{x}$ is conjugation by $a$. Then it is easy to show that the fixed point subgroup of $\pi$ is $\langle x, a\rangle$. By Lemma $4.4 \tau\langle x, a\rangle=\langle x, a\rangle$.

A similar argument can be applied to an arbitrary primitive element in $C_{x}$. Hence for every primitive $d \in C_{x}$

$$
\tau d=w_{d}(x, d)
$$

and $\tau$ preserves the subgroup $\langle x, d\rangle$. We then have

$$
\tau\langle x\rangle=\tau(\langle x, a\rangle \cap\langle x, b\rangle)=\langle x, a\rangle \cap\langle x, b\rangle=\langle x\rangle .
$$

Therefore $\tau x=x^{ \pm 1}$. In particular, the word $w_{a}(x, a)$ must have explicit occurrences of $a$. 
We claim now that the words $w_{d}$, where $d=a, b, a b$ have the same structure, that is any word $w_{d}(x, d)$ can be obtained from the word $w_{a}(x, a)$ by replacing occurrences of $a$ by $d$ :

$$
\left[w_{a}(x, a)\right]_{d}^{a}=w_{d}(x, d) .
$$

To prove this, it suffices to find in $\Pi$ automorphism of $F$ which takes $a$ to $b$ ( $a$ to $a b)$.

Let $\sigma_{1}$ and $\sigma_{1}^{\prime}$ be involutions in $\operatorname{Cen}(\varphi)$ such that $\sigma_{1}$ and $\sigma_{1}^{\prime}$ both fix the set $\{x\} \cup C$ pointwise and

$$
\begin{aligned}
\sigma_{1} a & =b^{-1}, & \sigma_{1}^{\prime} a & =a b, \\
\sigma_{1} b & =a^{-1}, & \sigma_{1}^{\prime} b & =b^{-1},
\end{aligned}
$$

Clearly, $\sigma_{1}$ and $\sigma_{1}^{\prime}$ are conjugate and $\pi_{1}=\sigma_{1} \sigma_{1}^{\prime}$ sends $a$ to $b$. Since $\tau$ and $\pi_{1}$ commute, we have

$$
\begin{aligned}
\tau a=w_{a}(x, a) & \Rightarrow \tau\left(\pi_{1} a\right)=w_{a}\left(\pi_{1} x, \pi_{1} a\right) \\
& \Rightarrow w_{b}(x, b)=w_{a}(x, b) .
\end{aligned}
$$

Thus, there is a reduced word $w$ in letters $x$ and, say, $t$ such that

$$
[w(x, t)]_{d}^{t}=w_{d}(x, d),
$$

where $d=a, b, a b$. We then have

$$
\tau(a b)=w(x, a b)=\tau(a) \tau(b)=w(x, a) w(x, b),
$$

and hence

$$
w(x, a b)=w(x, a) w(x, b) .
$$

Now we show that the word $w(x, t)$ has the form $x^{k} t x^{-k}$, where $k \in \mathrm{Z}$. Assume that $w(x, t)$ has the (possibly non-reduced) form such that

$$
x^{k_{1}} t^{l_{1}} x^{k_{2}} t^{l_{2}} \ldots x^{k_{m}} t^{l_{m}},
$$

where $k_{1}$ or $l_{m}$ could be equal to zero, whereas any other exponent is non-trivial. Then by (5.1)

$x^{k_{1}}(a b)^{l_{1}} x^{k_{2}}(a b)^{l_{2}} \ldots x^{k_{m}}(a b)^{l_{m}}=x^{k_{1}} a^{l_{1}} x^{k_{2}} a^{l_{2}} \ldots x^{k_{m}} a^{l_{m}} x^{k_{1}} b^{l_{1}} x^{k_{2}} b^{l_{2}} \ldots x^{k_{m}} b^{l_{m}}$.

The latter equality is evidently impossible when $m \geq 2$ and $l_{m} \neq 0$. Hence $l_{m}=0$ and $k_{m}=-k_{1}$. Even after this reduction (5.2) fails, if $m \geq 3$. Therefore

$$
x^{k_{1}}(a b)^{l_{1}} x^{-k_{1}}=x^{k_{1}} a^{l_{1}} b^{l_{1}} x^{-k_{1}},
$$

and we have

$$
(a b)^{l_{1}}=a^{l_{1}} b^{l_{1}} .
$$

Since $a$ and $b$ are independent, $l_{1}=1$. 
Summing up, we see that $\tau$ acts on $\mathcal{B}$ as follows

$$
\begin{aligned}
\tau x & =x^{\varepsilon}, \\
\tau a & =x^{k} a x^{-k}, \\
\tau b & =x^{k} b x^{-k}, \\
\tau c & =x^{k} c x^{-k}, \quad c \in C .
\end{aligned}
$$

where $\varepsilon= \pm 1$. In the case when $\varepsilon=-1, \tau$ is an involution, otherwise $\tau$ is conjugation by $x^{k}$. Conversely, every automorphism of $F$ of the form (5.3) is in $\operatorname{Cen}(\Pi)$.

Using Theorem 4.6, one can readily convert the hypotheses in Proposition 5.2 and Proposition 5.3 into first-order formulae. The proof of Theorem 5.1 is complete.

REMARK. In the case when $F$ has infinite rank the subgroup of all conjugations is first-order definable subgroup of $\operatorname{Aut}(F)$. Indeed, it is easy to see that every element in infinitely generated free group can be expressed as the product of two primitive elements. Therefore every conjugation in $\operatorname{Aut}(F)$ is the product of two conjugations by powers of primitive elements. This argument of course does not work for finitely generated free groups.

So all is now in readiness for a proof of the main result of the paper.

Theorem 5.4. Let $F$ be a non-abelian free group. Then the group $\operatorname{Aut}(F)$ is complete.

Proof. By Theorem 5.1 and Claim 1.2 the subgroup of $\operatorname{Aut}(F)$ generated by all conjugations by powers of primitive elements, namely the $\operatorname{subgroup} \operatorname{Inn}(F)$ of all conjugations is a characteristic subgroup of $\operatorname{Aut}(F)$. Therefore the group $\operatorname{Aut}(F)$ is complete (Proposition 1.1)

Theorem 5.5. The automorphism groups of free groups $F$ and $F^{\prime}$ are isomorphic if and only if $F \cong F^{\prime}$.

Proof. We can assume that both groups $F, F^{\prime}$ have ranks at least two. Any isomorphism from $\operatorname{Aut}(F)$ to $\operatorname{Aut}\left(F^{\prime}\right)$ preserves conjugations, and hence induces an isomorphism between $F$ and $F^{\prime}$.

\section{REFERENCES}

[1] R. Cohen, 'Classes of automorphisms of free group of infinite rank', Trans. Amer. Math. Soc., 177 (1973) 99-119.

[2] J. Dyer, E. Formanek, 'The automorphism group of a free group is complete', J. London Math. Soc., 11 (1975) 181-190.

[3] J. Dyer, G. P. Scott, 'Periodic automorphisms of free groups', Comm. Algebra0, 3 (1975) 195-201.

[4] E. Formanek, 'Characterizing a free group in its automorphism group', J. Algebra, 133 (1990) 424-432.

[5] W. Hodges, 'Model Theory', (University Press, Cambridge, 1993).

[6] D. G. Khramtsov, 'Completeness of groups of outer automorphisms of free groups', in Group-theoretic investigations (Russian), Akad. Nauk SSSR Ural. Otdel., Sverdlovsk, (1990) 128-143. 
[7] R. Lyndon, P. Schupp, 'Combinatorial group theory', (Springer-Verlag, Berlin, etc., 1977).

[8] W. Magnus, 'Untersuchungen über einige unendliche discontinuierliche Gruppen', Math. Ann., 105 (1931), 52-74.

[9] S. Meskin, 'Periodic automorphisms of the two-generator free group', Proc. Conf. Canberra 1973, (Lecture Notes in Math., 372, 494-498, Berlin, etc., Springer).

[10] W. Specht, Gruppentheorie (Berlin-Göttingen-Heidelberg, 1956).

[11] V. Tolstykh, 'Puissance et plénitude: interprétation des groupes d'automorphismes des groupes libres', Quatrième Colloque Franco-Touranien de Théorie des Modèles, MarseilleLumini 1997, Résumés des Conférences, Institut Girard Desargues, Université Claude Bernard, Lyon 1, 19.

Department of mathematics, Kemerovo State University, Krasnaja, 6, 650043 Kemerovo, Russia 